\title{
Two-Point One-Loop Fermionic Amplitudes in Constant Homogeneous Magnetic Field
}

\author{
Alexandra Dobrynina ${ }^{1, \star}$, Ilya Karabanov ${ }^{1, \star \star}$, Alexander Parkhomenko ${ }^{1, \star \star \star}$, and Lubov \\ Vassilevskaya ${ }^{2}$ \\ ${ }^{1}$ P. G. Demidov Yaroslavl State University, Yaroslavl, Russia \\ ${ }^{2}$ Fulda University of Applied Sciences, Fulda, Germany
}

\begin{abstract}
Two-point one-loop fermionic amplitudes modified by a constant homogeneous magnetic field are studied. In addition to the amplitudes resulted after an insertion of scalar, pseudoscalar, vector and axial-vector fermionic currents, we calculate similar amplitudes with the tensor and (pseudo)scalar vertices. The crossed-field limit of these amplitudes is presented. The tensor current is a fermionic part of the Pauli Lagrangian relevant for the electromagnetic interaction of fermions through the anomalous magnetic moment and its contribution to the photon polarization operator is briefly discussed.
\end{abstract}

\section{Introduction}

The general case of the two-point one-loop fermionic amplitudes modified by a constant homogeneous magnetic field was studied in Ref. [1]. The Lagrangian density of a local fermion interaction can be written in the form $[1,2]$ :

$$
\mathcal{L}_{\text {int }}(x)=\left[\bar{f}(x) \Gamma^{A} f(x)\right] J_{A}(x) \equiv j^{A}(x) J_{A}(x),
$$

where $f(x)$ is the quantum fermion field, $\Gamma_{A}$ is any of the $\gamma$-matrices from the standard set $\left\{1, \gamma_{5}, \gamma_{\mu}, \gamma_{\mu} \gamma_{5}, \sigma_{\mu \nu}\right\}$ [3], and $J_{A}(x)$ is a generalized electrically neutral current which can be, for example, a photon field, neutrino current, derivative of the axion field and etc. [the corresponding coupling constants are included into $J_{A}(x)$ ]. After the generalized currents are removed from a twopoint one-loop amplitude, it is reduced to a correlator of two fermionic currents (1) which can be written as follows [1,2]:

$$
\Pi_{A B}=\int d^{4} X \mathrm{e}^{-i(q X)} \operatorname{Sp}\left\{S_{\mathrm{F}}(-X) \Gamma_{A} S_{\mathrm{F}}(X) \Gamma_{B}\right\}
$$

where $q^{\mu}$ is the four-momentum carried by the generalized current and $S_{\mathrm{F}}(X)$ is the Lorentz-invariant part of an exact fermion propagator calculated in an external field background [4]. We assume the constant homogeneous magnetic field configuration for the external field. Among the existing representations of $S_{\mathrm{F}}(X)$ in this field, we accept the so-called Fock-Schwinger one [4, 5] in which the fermion

\footnotetext{
^e-mail: dobrynina@uniyar.ac.ru

$\star \star$ e-mail: karabanovilya@rambler.ru

$\star \star \star$ e-mail: parkh@uniyar.ac.ru
} 
propagator has an explicitly Lorentz-covariant form. Correlations, being under an influence of the magnetic field, among the scalar, pseudoscalar, vector and axial-vector currents in the Fock-Schwinger formalism were already studied $[1,2]$ but correlators with the tensor current $j_{\mu \nu}(x) \equiv\left[\bar{f}(x) \sigma_{\mu \nu} f(x)\right]$ and other currents $j_{A}(x)$ were not considered in this approach, except the pseudoscalar-tensor correlator calculated by us earlier [6].

In this paper, we present the propagator of a charged fermion in the constant homogeneous magnetic field in the Fock-Schwinger representation, show some selected results for the two-point correlation functions, find their crossed-field limits, and conclude with a discussion of an application of the amplitudes considered.

\section{Propagator in Constant Homogeneous Magnetic Field}

The general form of the charged fermion propagator in the Fock-Schwinger representation is known well $[4,5]$ :

$$
G_{\mathrm{F}}(x, y)=\mathrm{e}^{i \Omega(x, y)} S_{\mathrm{F}}(x-y),
$$

where $\Omega(x, y)$ is the Lorentz non-invariant phase. In the two-point one-loop amplitude, the phase factors of the two propagators cancel each other: $\Omega(x, y)+\Omega(y, x)=0$, and the Lorentz-invariant parts of the fermion propagators are left only [see Eq. (2)].

Let us consider a pure constant homogeneous magnetic field, $\mathbf{B}=(0,0, B)$. The corresponding four-potential can be written in the exactly Lorentz-covariant form, $A_{\mu}(x)=-F_{\mu \nu} x^{\nu} / 2$. Minkowski space filled with the constant homogeneous magnetic field is divided into two subspaces: the Euclidean one with the metric tensor $\Lambda_{\mu \nu}=(\varphi \varphi)_{\mu \nu}$, which is nothing else but the plane orthogonal to the field direction, and pseudo-Euclidean one with the metric tensor $\tilde{\Lambda}_{\mu \nu}=(\tilde{\varphi} \tilde{\varphi})_{\mu \nu}$. The metric tensor of Minkowski space is the difference of the subspaces' metrics, $g_{\mu \nu}=\tilde{\Lambda}_{\mu \nu}-\Lambda_{\mu v}$. In each subspace there is also the Levi-Civita symbol: $\varphi_{\mu \nu}$ and $\tilde{\varphi}_{\mu \nu}$, respectively. They are the dimensionless tensor of the external magnetic field $\varphi_{\mu \nu}=F_{\mu \nu} / B$ and its dual $\tilde{\varphi}_{\mu \nu}=\varepsilon_{\mu \nu \rho \sigma} \varphi^{\rho \sigma} / 2$, where $\varepsilon_{\mu \nu \rho \sigma}$ is the antisymmetric Levi-Civita symbol of the Minkowski space with the definition $\varepsilon^{0123}=1$ [3].

The Lorentz-invariant part of the fermion propagator is as follows [2]:

$$
\begin{aligned}
S_{\mathrm{F}}(X) & =-\frac{i \beta}{2(4 \pi)^{2}} \int_{0}^{\infty} \frac{d s}{s^{2}} \exp \left(-i\left[m_{f}^{2} s+\frac{1}{4 s}(X \widetilde{\Lambda} X)-\frac{\beta \cot (\beta s)}{4}(X \Lambda X)\right]\right) \\
& \times\left\{(X \widetilde{\Lambda} \gamma) \cot (\beta s)-i(X \widetilde{\varphi} \gamma) \gamma_{5}-\frac{\beta s}{\sin ^{2}(\beta s)}(X \Lambda \gamma)+m_{f} s[2 \cot (\beta s)+(\gamma \varphi \gamma)]\right\},
\end{aligned}
$$

where $\beta=e B\left|Q_{f}\right|$.

\section{Orthogonal Basis in Magnetic Field Background}

A correlator with a rank different from zero is convenient to decompose in some set of four independent vectors. In the magnetic field background, such an orthogonal basis naturally exists [2]:

$$
b_{\mu}^{(1)}=(q \varphi)_{\mu}, \quad b_{\mu}^{(2)}=(q \tilde{\varphi})_{\mu} \quad b_{\mu}^{(3)}=q^{2}(\Lambda q)_{\mu}-(q \Lambda q) q_{\mu}, \quad b_{\mu}^{(4)}=q_{\mu},
$$

and an arbitrary four-vector $a_{\mu}$ can be presented as follows:

$$
a_{\mu}=\sum_{i=1}^{4} a_{i} \frac{b_{\mu}^{(i)}}{\left(b^{(i)} b^{(i)}\right)}, \quad a_{i}=a^{\mu} b_{\mu}^{(i)}=\left(a b^{(i)}\right) .
$$


An arbitrary second-rank tensor $T_{\mu \nu}$ can be decomposed similarly:

$$
T_{\mu \nu}=\sum_{i, j=1}^{4} T_{i j} \frac{b_{\mu}^{(i)} b_{v}^{(j)}}{\left(b^{(i)} b^{(i)}\right)\left(b^{(j)} b^{(j)}\right)}, \quad T_{i j}=b_{\mu}^{(i)} T^{\mu v} b_{\nu}^{(j)}=\left(b^{(i)} T b^{(j)}\right) .
$$

This procedure can be easily extended to higher rank tensors.

\section{Correlators of Tensor Current in Magnetic Field}

The correlators containing the tensor current in combination with a fermionic current of other Lorentz structure are the second-, third- and fourth-rank tensors. If we restrict ourselves by the (pseudo)scalartensor correlator, the decomposition (7) for the second-rank tensor should be used. From six nontrivial coefficients in the basis decomposition, only three are independent. It is convenient to use the following double-integral representation for the coefficients in the decomposition:

$$
\begin{aligned}
\Pi_{i j}\left(q^{2}, q_{\perp}^{2}, \beta\right)= & \frac{1}{16 \pi^{2}} \int_{0}^{\infty} \frac{d t}{t} \int_{0}^{1} d u Y_{i j}\left(q^{2}, q_{\perp}^{2}, \beta ; t, u\right) \times \\
& \times \exp \left\{-i\left[m_{f}^{2} t-\frac{q_{\|}^{2}}{4} t\left(1-u^{2}\right)+q_{\perp}^{2} \frac{\cos (\beta t u)-\cos (\beta t)}{2 \beta \sin (\beta t)}\right]\right\},
\end{aligned}
$$

where the integration variables $t=s_{1}+s_{2}$ and $u=\left(s_{1}-s_{2}\right) /\left(s_{1}+s_{2}\right)$ are the combinations of two proper-time variables $s_{1}$ and $s_{2}$ entering the propagators. Note also the relation among the momenta squared: $q_{\|}^{2}=q^{2}+q_{\perp}^{2}$.

The integrands of the scalar-tensor correlator:

$$
\begin{aligned}
& Y_{13}^{(\mathrm{ST})}\left(q^{2}, q_{\perp}^{2}, \beta ; t, u\right)=\frac{1}{2} \beta q_{\|}^{2} q_{\perp}^{2}\left\{4\left[m_{f}^{2} t-i\right]+q^{2} t\left(1-u^{2}\right)+q_{\perp}^{2} t R(\beta ; t, u)\right\}, \\
& Y_{14}^{(\mathrm{ST})}\left(q^{2}, q_{\perp}^{2}, \beta ; t, u\right)=-\frac{1}{2} \beta q_{\perp}^{2}\left\{4\left[m_{f}^{2} t-i\right]+q_{\|}^{2} t R(\beta ; t, u)\right\} \\
& Y_{34}^{(\mathrm{ST})}\left(q^{2}, q_{\perp}^{2}, \beta ; t, u\right)=-\beta t q^{2} q_{\|}^{2} q_{\perp}^{2} \frac{\sin (\beta t u)}{\sin (\beta t)}[\cot (\beta t)-u \cot (\beta t u)],
\end{aligned}
$$

and pseudoscalar-tensor one ${ }^{1}$ :

$$
\begin{aligned}
& Y_{12}^{(\mathrm{PT})}\left(q^{2}, q_{\perp}^{2}, \beta ; t, u\right)=-i \beta t q_{\|}^{2} q_{\perp}^{2} \frac{\sin (\beta t u)}{\sin (\beta t)}[\cot (\beta t)-u \cot (\beta t u)], \\
& Y_{23}^{(\mathrm{PT})}\left(q^{2}, q_{\perp}^{2}, \beta ; t, u\right)=\frac{i}{2} \beta q_{\|}^{2} q_{\perp}^{2}\left\{4\left[m_{f}^{2} t+i\right]-q_{\|}^{2} t R(\beta ; t, u)\right\}, \\
& Y_{24}^{(\mathrm{PT})}\left(q^{2}, q_{\perp}^{2}, \beta ; t, u\right)=-\frac{i}{2} \beta q_{\|}^{2}\left\{4\left[m_{f}^{2} t+i\right]-q^{2} t\left(1-u^{2}\right)-q_{\perp}^{2} t R(\beta ; t, u)\right\} .
\end{aligned}
$$

To simplify the presentation, the auxiliary function is introduced:

$$
R(\beta ; t, u)=1-u^{2}+\frac{2}{\sin ^{2}(\beta t)}[\cos (\beta t) \cos (\beta t u)+u \sin (\beta t) \sin (\beta t u)-1] .
$$

\footnotetext{
${ }^{1}$ These integrands correct the ones presented in [6]
} 


\section{Correlators in the Crossed-Field Limit}

Correlators in an electromagnetic crossed field can be obtained from the ones calculated in the magnetic field after the pure field parameter $\beta_{\mathrm{F}}^{2}=e^{2} Q_{f}^{2} F_{\mu \nu} F^{\mu \nu} / 4$ is neglected. Quantities calculated in the crossed field are completely determined by the dynamical parameter $\chi_{f}^{2}=e^{2} Q_{f}^{2}(q F F q)=\beta^{2} q_{\perp}^{2}$. The crossed-field limit is valid for an ultrarelativistic particle moving in the direction transverse to the field strength in a relatively weak magnetic field. As basic vectors, it is convenient to use the following normalized orthogonal set:

$$
b_{\mu}^{(1)}=\frac{e Q_{f}}{\chi_{f}}(q F)_{\mu}, \quad b_{\mu}^{(2)}=\frac{e Q_{f}}{\chi_{f}}(q \tilde{F})_{\mu}, \quad b_{\mu}^{(3)}=\frac{e^{2} Q_{f}^{2}}{\chi_{f}^{2} \sqrt{q^{2}}}\left[q^{2}(F F q)_{\mu}-(q F F q) q_{\mu}\right], \quad b_{\mu}^{(4)}=\frac{q_{\mu}}{\sqrt{q^{2}}} .
$$

The coefficients in the tensor decomposition can be presented as double integrals:

$$
\Pi_{i j}\left(q^{2}, \chi_{f}\right)=\frac{1}{16 \pi^{2}} \int_{0}^{\infty} \frac{d t}{t} \int_{0}^{1} d u Y_{i j}\left(q^{2}, \chi_{f} ; t, u\right) \exp \left\{-i\left[\left(m_{f}^{2}-\frac{q^{2}}{4}\left(1-u^{2}\right)\right) t+\frac{1}{48} \chi_{f}^{2}\left(1-u^{2}\right)^{2} m_{f}^{6} t^{3}\right]\right\} .
$$

The integrands of the scalar-tensor correlator in the crossed-field Limit are as follows:

$$
\begin{aligned}
Y_{13}^{(\mathrm{ST})} & =\frac{\chi_{f}}{2 \sqrt{q^{2}}}\left\{4\left[m_{f}^{2} t-i\right]+q^{2} t\left(1-u^{2}\right)-\frac{1}{4} \chi_{f}^{2} t^{3}\left(1-u^{2}\right)^{2}\right\} \\
Y_{14}^{(\mathrm{ST})} & =\frac{\chi_{f}}{2 \sqrt{q^{2}}}\left\{4\left[m_{f}^{2} t-i\right]-\frac{1}{4} \chi_{f}^{2} t^{3}\left(1-u^{2}\right)^{2}\right\} \\
Y_{34}^{(\mathrm{ST})} & =\frac{1}{3} \chi_{f}^{2} t^{2} u\left(1-u^{2}\right)
\end{aligned}
$$

and of the pseudoscalar-tensor one are similar:

$$
\begin{aligned}
Y_{12}^{(\mathrm{PT})} & =\frac{i}{3} \chi_{f}^{2} t^{2} u\left(1-u^{2}\right) \\
Y_{23}^{(\mathrm{PT})} & =\frac{i \chi_{f}}{2 \sqrt{q^{2}}}\left\{4\left[m_{f}^{2} t+i\right]+\frac{1}{4} \chi_{f}^{2} t^{3}\left(1-u^{2}\right)^{2}\right\} \\
Y_{24}^{(\mathrm{PT})} & =\frac{i \chi_{f}}{2 \sqrt{q^{2}}}\left\{4\left[m_{f}^{2} t+i\right]+q^{2} t\left(1-u^{2}\right)+\frac{1}{4} \chi_{f}^{2} t^{3}\left(1-u^{2}\right)^{2}\right\} .
\end{aligned}
$$

In getting this limit, the expansion in $\beta$ of the function (15) is taken into account:

$$
R(\beta ; t, u) \simeq-\frac{1}{4} \beta^{2} t^{2}\left(1-u^{2}\right)^{2}-\frac{1}{72} \beta^{4} t^{4}\left(1-u^{2}\right)^{2}\left(3-u^{2}\right)-\cdots
$$

\section{Applications of Correlators}

Searches for physics beyond the Standard Model is the main stream of modern theoretical and experimental physics [7]. Models beyond the Standard Model can effectively modify the QED Lagrangian with an extra term called the Pauli Lagrangian density [8-10]:

$$
\mathcal{L}_{\mathrm{AMM}}(x)=\frac{\mu_{f}}{4}\left[\bar{f}(x) \sigma_{\mu \nu} f(x)\right] F^{\mu v}(x),
$$


where $\mu_{f}$ is the anomalous magnetic moment (AMM) of the fermion. The existence of (25) in the effective QED Lagrangian should also change the photon polarization operator which is nothing else but the correlator of two vector currents. If one makes a reasonable assumption about a smallness of $\mu_{f}$, the contribution linear in the fermion AMM, which is the vector-tensor correlator, is of importance. Its influence on the photon properties is under study and will be presented elsewhere [11].

The strong-magnetic-field formalism suggested by Loskutov and Skobelev [12, 13] allows to perform calculations very similar to the conventional approach developed for vacuum [3]. The disadvantage of this formalism is divergences which are appearing in two-point one-loop amplitudes. The most natural way to remove divergences is to consider the strong-field limit of the amplitudes calculated in the Fock-Schwinger formalism as it was demonstrated for the axion self-energy calculated in the magnetic field [14]. It is of interest to find the strong-field limit of all the two-point one-loop amplitudes and compare with ones obtained within the strong-magnetic-field formalism [12, 13].

\section{Conclusions}

Two-point one-loop fermionic amplitudes with the (pseudo)scalar and tensor vertices are considered. The influence of a constant homogeneous external magnetic field is taken into account exactly by using the exact propagators of a charged fermion in the Fock-Schwinger representation. The limit of the external electromagnetic crossed field is also obtained for the amplitudes considered.

\section{Acknowledgements}

A. D. and I. K. acknowledge support by RSF Grant No. 18-72-10070. A. P. is partially supported by the YSU Initiative Scientific Research Activity (Project No. AAAA-A16-116070610023-3).

\section{References}

[1] M.Yu. Borovkov, A.V. Kuznetsov, N.V. Mikheev, Phys. Atom. Nucl. 62, 1601 (1999), [Yad. Fiz. 62, 1714 (1999)]

[2] A.V. Kuznetsov, N.V. Mikheev, Electroweak Processes in External Active Media, Vol. 252 of Springer Tracts in Modern Physics (Springer-Verlag, New York, 2013)

[3] M.E. Peskin, D.V. Schroeder, An Introduction to Quantum Field Theory (Addison-Wesley, Reading, Massachusetts, 1995)

[4] C. Itzykson, J.B. Zuber, Quantum Field Theory (McGraw-Hill, New York, 1980)

[5] J.S. Schwinger, Phys. Rev. 82, 664 (1951)

[6] A. Dobrynina, I. Karabanov, A. Parkhomenko, L. Vassilevskaya, EPJ Web Conf. 158, 07001 (2017)

[7] M. Tanabashi et al. (Particle Data Group), Phys. Rev. D98, 030001 (2018)

[8] P.M. Lavrov, J. Phys. A18, 3455 (1985)

[9] H.K. Lee, Y. Yoon, JHEP 03, 086 (2007), hep-th/0611134

[10] A. Barducci, R. Giachetti, G. Pettini, Eur. Phys. J. C70, 445 (2010), 1006. 3871

[11] L.A. Vasilevskaya, A.A. Dobrynina, I.V. Karabanov, A.Ya. Parkhomenko, in preparation (2018)

[12] V.V. Skobelev, Russ. Phys. J. 18, 1481 (1975)

[13] Y.M. Loskutov, V.V. Skobelev, Phys. Lett. A56, 151 (1976)

[14] L.A. Vasilevskaya, N.V. Mikheev, A.Ya. Parkhomenko, Phys. Atom. Nucl. 64, 294 (2001), [Yad. Fiz. 64, 342 (2001)] 